15

\title{
Особенности переориентации поля директора и эволюции ЯМР-спектров под действием скрещенных электрического и магнитного полей
}

\author{
(C) A.B. Захаров
}

Институт проблем машиноведения РАН, Санкт-Петербург, Россия

E-mail: alexandre.zakharov@yahoo.ca

(Поступила в Редакцию 24 мая 2016 г.)

\begin{abstract}
Предложено теоретическое описание новых режимов переориентации поля директора $\hat{\mathbf{n}}$ и эволюции ЯМР-спектра $I(v)$ нематического жидкого кристалла (ЖК), образованного молекулами дейтерированного 4-n-пентил-4'-цианобифенила, инкапсулированного в прямоугольную ЖК-ячейку, под действием сильных скрещенных электрического $\mathbf{E}$ и магнитного В полей, направленных под углом $\alpha$ друг к другу. Численные расчеты, выполненные в рамках нелинейного обобщения классической теории Эриксена-Лесли, показали, что при определенных соотношениях сил и моментов, действующих на единицу объема ЖК-фазы, в процессе переориентации $\hat{\mathbf{n}}$ могут возникнуть переходные периодические структуры, если соответствующая мода искажения обладает наибыстрейшим откликом и таким образом подавляет все остальные моды, в том числе и однородные. Показано, что возникновение этих периодических структур ведет к уменьшению времени переориентации поля директора.
\end{abstract}

Работа выполнена при финансовой поддержке РФФИ (грант № 16-02-00041a).

\section{1. Введение}

Основным параметром, характеризующим качество жидкокристаллического (ЖК) дисплея, является время $\tau_{O N} \sim \frac{\gamma_{1}}{E^{2}}$ необходимое для переориентации поля директора $\hat{\mathbf{n}}$ под действием внешнего электрического поля $\mathbf{E}[1]$. Если это поле направлено, например, поперек ЖК-ячейки, то его величина должна превышать критическое значение [1] $E_{t h}=\frac{\pi}{d} \sqrt{\frac{K_{3}}{\epsilon_{0} \epsilon_{a}}}$. Здесь $\gamma_{1}$ и $K_{3}$-коэффициенты вращательной вязкости (КВВ) и продольной упругости ЖК-материала соответственно, $d-$ толщина ЖК-ячейки, $\epsilon_{0}$ - диэлектрическая проницаемость вакуума и $\epsilon_{a}$ - диэлектрическая анизотропия ЖК-материала. Таким образом, время переориентации директора $\tau_{O N}$ прямо пропорционально величине $\gamma_{1}$ и обратно пропорционально квадрату величины электрического поля $E$. Температурная зависимость $\gamma_{1}(T)$ достаточно хорошо изучена для большинства нематиков [2], за исключением области температур вблизи фазового перехода нематик-смектик $A(\mathrm{NA}), T_{N A}[3,4]$, где $\lim _{T \rightarrow T_{N A}} \gamma_{1}(T) \rightarrow \infty$. Такое поведение $\gamma_{1}(T)$ предполагает, что и величина $\tau_{O N}\left(T \rightarrow T_{N A}\right) \rightarrow \infty$. Расходимость $\lim _{T \rightarrow T_{N A}} \tau_{O N} \rightarrow \infty$, когда значения температуры отличаются от $T_{N A}$ на несколько десятков $m K$, была экспериментально подтверждена методами ЯМР-спектроскопии [5]. Было показано, что величина $\tau_{O N}\left(T \rightarrow T_{N A}\right)$ возрастает на несколько порядков и достигает величины $\sim 140 h$ для случая ЖК-фазы, образованной молекулами 4-n-нонил-4'-цианобифенила (9ЦБ).

В то же время, в случае $E \gg E_{t h}$ в процессе переориентации поля директора $\hat{\mathbf{n}}$ могут возникнуть переходные периодические структуры, если соответствующая мода искажения обладает наибыстрейшим откликом и таким образом подавляет все остальные моды, в том числе и однородные [6]. При периодическом искажении ЖК-фазы появляется сдвиговая вязкость, уменьшающая общую эффективную вращательную вязкость, связанную с переориентацией поля директора. Возникающие при этом вращающиеся домены способствуют уменьшению эффективной вязкости, характеризующей скорость диссипации энергии, и тем самым создают более выгодные, по сравнению с однородным поворотом, режимы переориентации поля директора. Все это, в конечном счете, ведет к уменьшению времени переориентации поля директора $\tau_{O N}$.

Методы ЯМР-спектроскопии позволяют измерить времена переориентации поля директора в ЖК-ячейках [7]. Это достигается тем, что ЖК-образец вначале ориентируется сильным магнитным полем В с соответствующим расщеплением квадрупольного спектра $\Delta \bar{\nu}_{0}$. Если в какой-то момент времени включить сильное поперечное электрическое поле E (turn-on процесс), то это ведет к убыванию величины расщепления квадрупольного спектра $\Delta \bar{\nu}$. При этом величина $\Delta \bar{\nu}(t) / \Delta \bar{\nu}_{0}=P_{2}(\cos \theta(t))$ связана с углом отклонения $\theta(t)$ поля директора от направления магнитного поля В (см. рис. 1). Здесь $P_{2}(x)$ полином Лежандра второго порядка. Таким образом, ЯМР-спектроскопия позволяет проследить эволюцию угла $\theta(t)$ от его начального значения $\theta_{0}$ до конечного $\theta_{\infty}$. Располагая зависимостью $\theta(t)$ (или $\Delta \bar{v}(t) / \Delta \bar{v}_{0}$ ) от времени, можно определить время $\tau_{O N}$, необходимое для переориентации поля директора, которая характеризуется уменьшением $\Delta \bar{v}$ в два раза относительно $\Delta \bar{v}_{0}$.

Теоретическое описание процесса переориентации поля директора $\hat{\mathbf{n}}$, описываемое углом $\theta(t)$, под действием 


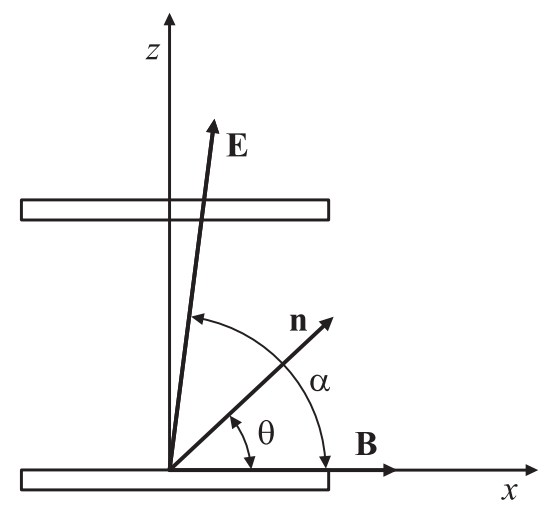

Рис. 1. Система координат, используемая при вычислениях. Орт $\hat{\mathbf{i}}$ направлен параллельно, а орт $\hat{\mathbf{k}}$ перпендикулярно к направлению вектора магнитного поля В. Вектор электрического поля $\mathbf{E}$ и вектор поля директора $\hat{\mathbf{n}}$ направлены под углом $\alpha$ и $\theta$ к направлению вектора В соответственно.

скрещенных электрического и магнитного полей, будет дано в рамках нелинейного обобщения классической теории Эриксена-Лесли [8,9]. Располагая временными зависимостями угла $\theta(t)$, соответствующего режимам включения (turn-on) и выключения (turn-off) внешнего электрического поля, будут рассчитаны ЯМР-спектры и дан сравнительный анализ расчетных и экспериментальных результатов для ЯМР-спектров.

\section{2. Основные уравнения}

Рассмотрим ЖК-систему образованную молекулами дейтерированного 4-n-пентил-4' -цианобифенила $\left(5 Ц\right.$ Ц- $\left.d_{2}\right)$ (см. рис. 2), помещенную в длинную прямоугольную ЖК-ячейку с размерами $2 L$ и $2 d(L \gg d)$, ограниченную твердыми горизонтальными и вертикальными поверхностями. Допустим, что директор планарно и жестко ориентирован на горизонтальных и гомеотропно на вертикальных ограничивающих поверхностях.

Выберем систему координат таким образом, чтобы она отсчитывалась от центра ЖК-ячейки, и при этом ось $x$ и орт $\hat{\mathbf{i}}$ совпадали с направлением директора на нижней горизонтальной поверхности $\left(\hat{\mathbf{i}} \| \hat{\mathbf{n}}_{\mathrm{z}=-\mathrm{d}}\right)$, в то время как ось $z$ и орт $\hat{\mathbf{k}}$ направлены ортогонально $\left(\hat{\mathbf{k}} \perp \hat{\mathbf{n}}_{\mathrm{z}=-\mathrm{d}}\right)$, а орт $\hat{\mathbf{j}}=\hat{\mathbf{k}} \times \hat{\mathbf{i}}$ (см. рис. 1$)$. Направим сильное магнитное поле $\mathbf{B}(\sim 7 T)$ параллельно горизонтальным поверхностям. Таким образом, в начальный момент времени мы имеем дело<smiles>[2H]C(C)(C)CCC</smiles>

Рис. 2. 5ЦБ- $d_{2}$-молекула. с планарно и однородно ориентированным ЖК-образцом, образованным молекулами $5 Ц$ Ц- $d_{2}$. Пусть этому состоянию соответствует расщепление квадрупольного ЯМР-спектра $\Delta \bar{v}$ [7]. Допустим, что в момент $t=0$ было включено сильное электрическое поле $\mathbf{E}=E_{x} \hat{\mathbf{i}}+E_{z} \hat{\mathbf{k}}=E(z) \cos \alpha \hat{\mathbf{i}}+E(z) \sin \alpha \hat{\mathbf{k}}$ направленное под углом $\alpha\left(\sim \frac{\pi}{2}\right)$ к направлению магнитного поля $\mathbf{B}$. Возникающая вследствие этого переориентация поля директора $\hat{\mathbf{n}}=n_{x} \hat{\mathbf{i}}+n_{z} \hat{\mathbf{k}}=\cos \theta(x, z, t) \hat{\mathbf{i}}+\sin \theta(x, z, t) \hat{\mathbf{k}}$ осуществлялась в плоскости $x O z$, образованной векторами $\mathbf{E}$ и В. Здесь $\theta-$ угол образованный вектором $\hat{\mathbf{n}}$ и ортом $\hat{\mathbf{i}}$. Эта переориентация поля директора сопровождалась убыванием величины расщепления квадрупольного спектра $\Delta \bar{v}$. При этом величина $\Delta \bar{v}(t) / \Delta \bar{v}_{0}$ связана с углом $\theta(t)$ отклонения директора $\hat{\mathbf{n}}$ от направления магнитного поля В соотношением $P_{2}(\cos \theta(t))=\Delta \bar{v}(t) / \Delta \bar{v}_{0}$. Располагая эволюцией угла $\theta(t)$ от его начального значения $\theta_{0}$ до конечного $\theta_{\infty}$, мы можем рассчитать эволюцию дейтеронного ЯМР-спектра $I(v)$ по формуле [7]

$$
I(v)=\int_{-\pi}^{\pi} f(\theta) I(v, \theta) \sin \theta d \theta,
$$

где $f(\theta)$ - плотность вероятности ориентации поля директора $\hat{\mathbf{n}}$, характеризующаяся углом $\theta(t)$, а функция

$$
I(v, \theta)=\sum_{ \pm} \frac{1}{\sqrt{2 \pi} \sigma(\theta)} \exp \left[-\frac{\left(v-v_{ \pm}(\theta)\right)^{2}}{2 \sigma^{2}(\theta)}\right]
$$

выбрана в Гауссовой форме, где угловая зависимость резонансной частоты $v_{ \pm}(\theta)$ имеет вид

$$
\nu_{ \pm}(\theta)=v_{0} \pm \frac{\Delta \bar{\nu}_{0}}{2} P_{2}(\cos \theta(t))
$$

Здесь $v_{0}-$ резонансная частота Зеемана [7], в то время как ширина линии $\sigma(\theta)$ может быть записана в виде [7]

$$
\sigma(\theta)=\sigma_{0}+\sigma_{2} P_{2}(\cos \theta)+\sigma_{4} P_{4}(\cos \theta),
$$

где $P_{2 L}$ - полином Лежандра $2 L$-порядка. В дальнейшем будем использовать нормированную плотность вероятности [10]

$$
f(\theta)=\frac{\xi}{2\left[\xi-(\xi-1) \cos ^{2} \theta\right]^{3 / 2}},
$$

где параметр $\xi$ варьируется в пределах от 1, соответствущей изотропной фазе, и до $\xi \rightarrow \infty$, когда $f(\theta)$ представляет собой $\delta$-функцию Дирака.

Таким образом, чтобы рассчитать эволюцию ЯМР-спектра $I(v)$ в процессе переориентации поля директора под действием скрещенных электрического и магнитного полей, прежде всего необходимо рассчитать эволюцию угла $\theta(t)$. В нашем случае эволюция угла $\theta(x, z, t)$ к его равновесному распределению $\theta_{e q}(x, z)$ может быть рассчитана с помощью уравнения баланса угловых моментов, действующих на единичный объем ЖК-фазы $\mathbf{T}_{\text {elast }}+\mathbf{T}_{\text {vis }}+\mathbf{T}_{\text {el }}+\mathbf{T}_{\text {mag }}+\mathbf{T}_{\text {flex }}=0$ и закона 
сохранения $\quad$ заряда $\quad \nabla \cdot \mathbf{D}=0 . \quad$ Здесь $\quad \mathbf{T}_{\text {elast }}=T_{\text {elast }} \hat{\mathbf{j}}=$ $=\frac{\delta \mathscr{W}_{\mathrm{F}}}{\delta \hat{\mathbf{n}}} \times \hat{\mathbf{n}}-$ упругий, $\mathbf{T}_{\text {vis }}=T_{\text {vi }} \hat{\mathbf{j}}=\frac{\delta \mathscr{R}^{\mathrm{vis}}}{\delta \hat{\mathbf{n}}_{t}} \times \hat{\mathbf{n}}-$ вязкий, $\mathbf{T}_{\mathrm{el}}=T_{\mathrm{el}} \hat{\mathbf{j}}=\frac{\delta \psi_{\mathrm{el}}}{\delta \hat{\mathbf{n}}} \times \hat{\mathbf{n}}-$ электрический, $\mathbf{T}_{\mathrm{mag}}=T_{\mathrm{mag}} \hat{\mathbf{j}}=$ $=\frac{\delta \psi_{\mathrm{mag}}}{\delta \hat{\mathbf{n}}} \times \hat{\mathbf{n}}-$ магнитный и $\mathbf{T}_{\text {flex }}=T_{\text {flex }} \hat{\mathbf{j}}=\frac{\delta \psi_{\text {flex }}}{\delta \hat{\mathbf{n}}} \times \hat{\mathbf{n}}-$ флексоэлектрический вклады в баланс угловых моментов, в то время как $\mathscr{W}_{\mathrm{F}}=\frac{1}{2}\left[K_{1}(\nabla \cdot \hat{\mathbf{n}})^{2}+\right.$ $\left.+K_{3}(\hat{\mathbf{n}} \times \nabla \times \hat{\mathbf{n}})^{2}\right] \quad$ - плотность упругой энергии приходящейся на единицу объема ЖК-фазы, $\psi_{\mathrm{el}}=-\frac{1}{2} \epsilon_{0} \epsilon_{a}(\hat{\mathbf{n}} \cdot \mathbf{E})^{2} \quad$ и $\quad \psi_{\mathrm{mag}}=-\frac{1}{2} \frac{\chi_{a}}{\mu_{0}}(\hat{\mathbf{n}} \cdot \mathbf{B})^{2}-$ плотности энергий электрического и магнитного полей, $\psi_{\text {flex }}=-\mathbf{P} \cdot \mathbf{E}-$ плотность энергии, создаваемая флексоэлектрическими силами, и $\mathscr{R}_{\mathrm{vis}}=\gamma_{1} \hat{\mathbf{n}}_{, t}=$ $=\gamma_{1}\left(n_{x, t}^{2}+n_{z, t}^{2}\right)-$ вязкий вклад в полную функцию Рэлея $\mathscr{R}$. Здесь $\hat{\mathbf{n}}_{, t}=\frac{d \hat{\mathbf{n}}}{d t}-$ материальная производная вектора $\hat{\mathbf{n}}, K_{1}$ и $K_{3}-$ коэффициенты упругости Франка, соответствующие поперечному и продольному изгибам, D - вектор электрической индукции, а вектор поляризации $\mathbf{P}$ равен $e_{1} \hat{\mathbf{n}}(\nabla \cdot \hat{\mathbf{n}})+e_{3}(\nabla \times \hat{\mathbf{n}} \times \hat{\mathbf{n}})$, где $e_{1}$ и $e_{3}-$ флексоэлектрические коэффициенты ЖК-системы, $\chi_{a}$ - магнитная анизотропия ЖК-системы, и $\mu_{0}$ - магнитная постоянная.

Следует отметить, что в процессе переориентации $\hat{\mathbf{n}}$ под действием сильных скрещенных электрического и магнитного полей в ЖК-ячейке возникает течение ЖК-материала, оказывающее незначительное влияние на эволюцию угла $\theta(x, z, t)$ к его равновесному распределению, которым мы в дальнейшем пренебрегаем [11].

Для случая двумерной ЖК-системы безразмерное уравнение баланса моментов, действующих на единицу объема ЖК-фазы, может быть записано в виде [11]

$$
\begin{gathered}
\theta_{, \tau}=\delta_{1}\left[\Delta_{1} \theta_{, x x}+\Delta_{2} \theta_{, z z}+\Delta_{3}\left(-2 \theta_{, x z}+\theta_{, x}^{2}-\theta_{, z}^{2}\right)+\Delta_{4} \theta_{, x} \theta_{, z}\right] \\
+\frac{1}{2}\left[f^{2}(\theta) \sin 2(\alpha-\theta)-\delta_{2} \sin 2 \theta\right]-\delta_{3} f_{, z}(\theta) \sin 2 \theta \sin \alpha
\end{gathered}
$$

где

$$
\begin{gathered}
\Delta_{1}=\sin ^{2} \theta+K_{31} \cos ^{2} \theta, \quad \Delta_{2}=\cos ^{2} \theta+K_{31} \sin ^{2} \theta, \\
\Delta_{3}=\frac{\left(1-K_{31}\right)}{2} \sin 2 \theta, \quad \Delta_{4}=\left(K_{31}-1\right) \cos 2 \theta, \\
f(\theta)=\frac{C-\delta_{3} \sin 2 \theta \theta_{, z}}{\epsilon_{\perp} / \epsilon_{a}+\sin ^{2} \theta} \\
C=\left[\int_{-1}^{+1}\left(\epsilon_{\perp} / \epsilon_{a}+\sin ^{2} \theta\right)^{-1} d z\right]^{-1}
\end{gathered}
$$

- функции системы, в то время как

$\delta_{1}=\frac{4 K_{1}}{\epsilon_{0} \epsilon_{a} U^{2}}, \quad \delta_{2}=\frac{4 \chi_{a} B^{2} d^{2}}{\mu_{0} \epsilon_{0} \epsilon_{a} U^{2}}, \quad \delta_{3}=\frac{e_{1}+e_{3}}{\epsilon_{0} \epsilon_{a} U} \quad$ и $\quad K_{31}=\frac{K_{3}}{K_{1}}$

- четыре параметра ЖК-системы. Здесь и далее $\bar{x}=x / d, \quad \bar{z}=z / d$ и $\tau=\frac{\epsilon_{0} \epsilon_{a}}{\gamma_{1}^{2}}\left(\frac{U}{2 d}\right)^{2}-$ безразмерные пространственные переменные и безразмерное время соответственно, причем в дальнейшем верхняя черта над пространственными переменными будет опущена.
В свою очередь, последнее уравнение (6) должно быть дополнено уравнением сохранения заряда $\nabla \cdot \mathbf{D}=0$, где $\mathbf{D}(z)$ - поле вектора электрической индукции, имеющее в нашем случае только $z$-компоненту, связанную с электрическим полем соотношением $[6,11]$

$$
\begin{gathered}
\frac{\partial}{\partial z}\left[\left(\frac{\epsilon_{\perp}}{\epsilon_{a}}+\sin ^{2} \theta\right) \bar{E}(z)+\delta_{3} \theta_{, z} \sin 2 \theta\right]=0, \\
\int_{-1}^{1} \bar{E}(z) d z=1,
\end{gathered}
$$

где функция $\bar{E}(z)=\frac{2 d E(z)}{U}$ описывает безразмерное электрическое поле, а $U=2 d E-$ величина напряжения, приложенного поперек ЖК-ячейки. Уравнение баланса моментов, записанное на границах ЖК-ячейки, дает нам граничные условия для угла $\theta$. Допустим, что директор планарно и жестко ориентирован на горизонтальных, и гомеотропно на вертикальных ограничивающих поверхностях. В этом случае граничные условия для угла $\theta$ принимают вид

$$
\theta_{-10<x<10, z= \pm 1}=\theta_{x= \pm 10,-1<z<1}=0 .
$$

Для того, чтобы наблюдать формирование пространственно периодической структуры в ЖК-ячейке под действием скрещенных магнитного и электрического полей, мы рассмотрим начальное условие для угла $\theta$ в виде $[11,12]$

$$
\theta(x, z, 0)=\theta_{0} \cos \left(q_{z} z\right) \cos \left(q_{x} x\right)
$$

где $\theta_{0}-$ амплитуда, а $q_{x}$ и $q_{z}-$ волновые числа соответствующей Фурье моды, которые имеют вид [6]

$$
\begin{array}{ll}
q_{x}=\frac{\pi}{20}(2 k+1), & k=0,1,2, \ldots, \\
q_{z}=\frac{\pi}{20}(2 l+1), & l=0,1,2, \ldots .
\end{array}
$$

В свою очередь безразмерные волновые числа $q_{x}$ и $q_{z}$ определяются из условия минимума полной энергии $W=W_{\text {elast }}+W_{\text {el }}$, где

$$
\begin{aligned}
& \frac{2}{\delta_{1}} W_{\text {elast }}=\int d x \int d z\left[\left(\left(\theta_{e q}\right)_{, x}^{2}+\left(\theta_{e q}\right)_{, z}^{2}\right)\right. \\
& \left.\quad \times\left(\sin ^{2} \theta_{e q}+K_{31} \cos ^{2} \theta_{e q}\right)\right] \\
& \quad+\int d x \int d z\left(K_{31}-1\right) \sin 2 \theta_{e q}\left(\theta_{e q}\right)_{, x}\left(\theta_{e q}\right)_{, z}
\end{aligned}
$$

есть вклад упругих сил, а

$$
W_{\mathrm{el}}=-\int d x \int d z\left[E\left(\theta_{e q}\right) \cos ^{2}\left(\theta_{e q}-\alpha\right)+\delta_{3} \cos ^{2} \theta\right]
$$

есть вклад электромагнитных сил в общую энергию $W$ соответственно. Таким образом, система уравнений (6)-(7), дополненная граничными (8) и начальным (9) условиями, образует самосогласованную систему нелинейных дифференциальных уравнений в частных 
производных, описывающих эволюцию поля директора $\hat{\mathbf{n}}$ к его равновесному распределению по всему объему микрометровой ЖК-ячейки под действием сильного электрического поля $\mathbf{E}$, направленного под углом $\alpha$ к магнитному полю В. В случае формирования пространственно периодической структуры, значения волновых чисел $q_{x}$ и $q_{z}$ определяются из условия минимальности полной энергии.

\section{3. Эволюция поля директора и ЯМР-спектров под действием скрещенных электрического и магнитного полей}

Процесс переориентации поля директора и эволюции ЯМР-спектров в ЖК-фазе, образованной молекулами дейтерированного цианобифенила 5ЦБ- $d_{2}$ (см. рис. 2), был исследован для случая ЖК-ячейки толщиной $2 d=194.7 \mu \mathrm{m}(L / d=10)$, при температуре $300 \mathrm{~K}$ и плотности $10^{3} \mathrm{~kg} / \mathrm{m}^{3}$. Здесь $2 L-$ длина ЖК-ячейки, а значения коэффициентов упругости $K_{1}$ и $K_{3}$ равны $9.5 \mathrm{pN}$ и $13.8 \mathrm{pN}$ [13] соответственно, в то время как значение диэлектрической анизотропии $\epsilon_{a}$ равно 11.5 [14], a $\gamma_{1}=0.135$ Pas [2]. Величины напряжения $U$, приложенного поперек ЖК-ячейки и магнитного поля $\mathbf{B}$ были выбраны равными $U=200 \mathrm{~V}$ и $B=7.08 \mathrm{~T}$, что соответствует значениям этих величин используемых в ЯМР-спектроскопических измерениях [7,12]. Значение магнитной анизотропии $\chi_{a}$ равно $1.17 \times 10^{-6}$, в то время как значения флексоэлектрических коэффициентов $e_{1}$ и $e_{3}$ были выбраны равными $-11.6 \mathrm{pC} / \mathrm{m}$ и $-4.3 \mathrm{pC} / \mathrm{m}$ [15] соответственно. Таким образом, $\delta$-параметры, перечисленные выше, принимают следующие значения: $\delta_{1}=8.6 \cdot 10^{-6}, \delta_{2}=0.424, \delta_{3}=-9 \cdot 10^{-4}$, в то время как параметр $K_{31}$ равен 1.17. Следует отметить, что величина порогового напряжения в нашем случае равна $E_{t h} \sim 1.05 \cdot 10^{4} \mathrm{~V} / \mathrm{m}$, так что $E \sim 100 E_{t h}$.

Когда сильное электрическое поле $\mathbf{E}\left(E \sim 100 E_{t h}\right.$, $\sim 1 \mathrm{~V} / \mu \mathrm{m})$, включенное в момент времени $\tau=0$, направлено под углом $\alpha \sim \frac{\pi}{2}$ к направлению магнитного поля В $(\sim 7.08 \mathrm{~T})$, то планарно и однородно ориентированный нематический образец, образованный дейтерированными молекулами 5ЦБ- $d_{2}$, стремится переориентироваться в направлении вектора Е. На рис. 3 и 4 представлены результаты расчета эволюции угла $\theta(x, z=0, \tau)$ к его равновесному распределению $\theta_{e q}(x, z=0)$ вдоль оси $x \in[-10,10]$, в режиме turn-on, которое достигается спустя $\tau_{R}$ единиц безразмерного времени, после включения электрического поля $E \sim 100 E_{t h}$. При этом система нелинейных дифференциальных уравнений в частных производных (6)-(7) совместно с граничным (8) и начальным (9) условиями была решена методом релаксаций [16], а критерий сходимости итерационной процедуры был выбран равным $\epsilon=\left|\left(\theta_{(m+1)}-\theta_{(m)}\right) / \theta_{(m)}\right| \sim 10^{-4}$, и итерационная процедура продолжалась до достижения заданной

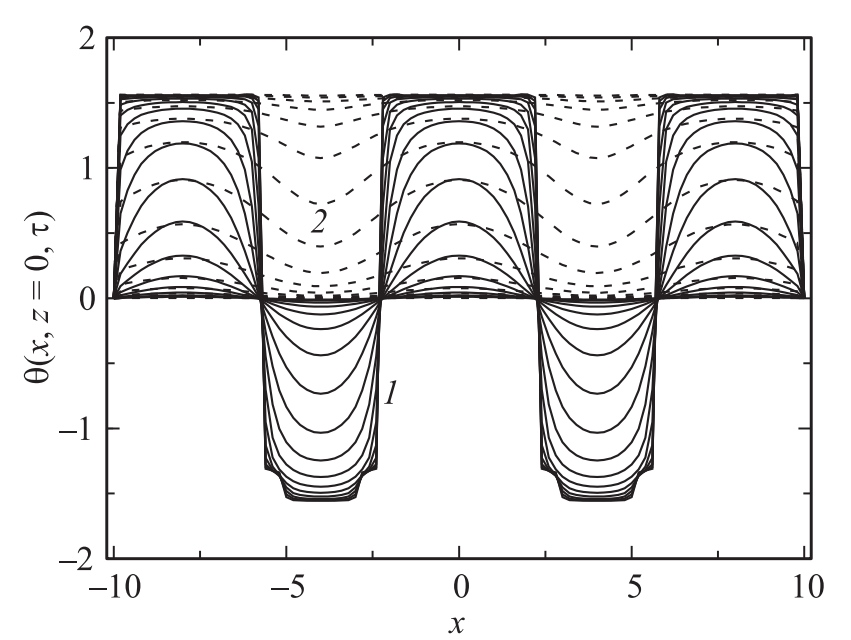

Рис. 3. Эволюция распределения угла $\theta(x, z=0, \tau)$ вдоль оси $x \in[-10,10]$ к его равновесному распределению $\theta_{e q}(x, z=0)=\theta_{e q}\left(x, z=0, \tau=\tau_{O N}^{(1)}=20\right)$, при $\alpha=1.57$ и двух значениях амплитуды $\theta_{0}=0.01$ (сплошные кривые) и 0.001 (штриховые кривые) соответственно.

точности. Так, на рис. 3 представлены результаты расчета эволюции угла $\theta(x, z=0, \tau)$ к его равновесному распределению $\theta_{e q}(x, z=0)$ вдоль оси $x \in[-10,10]$, в режиме turn-on, в течение $\tau_{R}=20$ единиц безразмерного времени, отсчитанного с момента включения электрического поля $E \sim 100 E_{t h}$. Вначале значение угла $\alpha$ было выбрано равным $1.57\left(\sim 89.96^{\circ}\right)$, при двух значениях амплитуды $\theta_{0}=0.01\left(\sim 1.1^{\circ}\right)$ и $0.001\left(\sim 0.1^{\circ}\right)$. Основной результат этих расчетов заключается в том, что при фиксированном значении угла $\alpha=1.57$ существует пороговое значение амплитуды $\theta_{0}^{\text {th }}$, выше которого в процессе переориентации поля директора $\hat{\mathbf{n}}$ возникают переходные периодические структуры, формирующиеся как вдоль оси $x$, так и вдоль оси $z$. Так, на рис. 3 (сплошные кривые), при $\theta_{0}=0.01$ и $z=0$, показано формирование периодической структуры вдоль оси $x \in[-10,10]$, которая характеризуется узлами в точках $x= \pm 2.33, \pm 5.83$ и \pm 9.83 и равновесное распределение $\theta_{e q}^{(1)}(x, z=0)$ достигается спустя $\tau_{O N} \sim 20$ единиц безразмерного времени $(\sim 25.4 \mu \mathrm{m})$. В то же время при значении амплитуды $\theta_{0}=0.001$, т.е. в десять раз меньше, чем в первом случае, периодическая структура в процессе переориентации поля директора не возникает. В этом случае процесс переориентации $\hat{\mathbf{n}}$ реализуется в форме монодомена, с осциллирующим профилем $\theta(x, z=0, \tau)$ (см. рис. 3, штриховые кривые) направленным в положительном направлении и заканчивающимся равновесным распределением $\theta_{e q}^{(2)}(x, z=0)$, которое достигалось спустя $\tau_{O N} \sim 25$ единиц безразмерного времени $(\sim 31.75 \mu \mathrm{m})$. Физически это означает, что в первом случае с $\alpha=1.57$ и $\theta_{0}=0.01$ величины электрического поля $E \sim 100 E_{t h}$ достаточно для формирования переходной периодической структуры, которая создает более выгодный и более быстрый по сравнению с однородным поворотом режим переориентации поля 


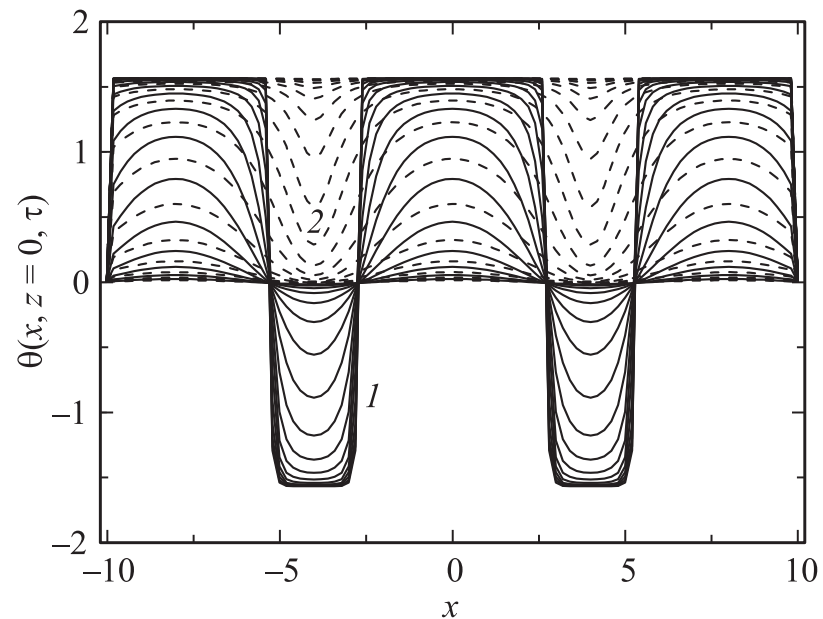

Pис. 4. То же, что на рис. 3 , но при $\alpha=1.565$ и двух значениях амплитуды $\theta_{0}=0.02$ (сплошные кривые) и 0.01 (штриховые кривые) соответственно.

директора. По мере уменьшения величины угла $\alpha$, с $1.57\left(\sim 89.96^{\circ}\right)$ до $1.565\left(\sim 88.81^{\circ}\right)$, формирование периодической структуры вдоль оси $x \in[-10,10]$ возможно лишь при значениях амплитуды $\theta_{0} \geq 0.02\left(\sim 1.2^{\circ}\right)$. На рис. 4 (сплошные кривые), при $\bar{\theta}_{0}=0.02$ и $z=0$, показано формирование периодической структуры вдоль оси $x \in[-10,10]$, которая характеризуется узлами в точках $x= \pm 2.6, \pm 5.3$ и \pm 9.93 , и равновесное распределение $\theta_{e q}^{(3)}(x, z=0)$ достигалось спустя $\tau_{O N} \sim 20$ единиц безразмерного времени $(\sim 25.4 \mu \mathrm{m})$. В то же время при значении амплитуды $\theta_{0}=0.01$ периодическая структура в процессе эволюции угла $\theta(x, z=0, \tau)$ не возникает. В этом случае процесс переориентации поля директора n реализуется в форме монодомена, с осциллирующим профилем $\theta(x, z=0, \tau)$ (см. рис. 4, штриховые кривые) направленным в положительном направлении и заканчивающимся равновесным распределением $\theta_{e q}^{(2)}(x, z=0)$, которое достигалось спустя $\tau_{O N} \sim 21$ единиц безразмерного времени $(\sim 26.7 \mu \mathrm{m})$. Таким образом установлено, что по мере уменьшения величины угла $\alpha$ с 1.57 до 1.565 пороговое значение амплитуды $\theta_{0}^{\text {th }}$ возрастает в 2 раза, до величины 0.02 , а значения волновых чисел $q_{x}$ и $q_{z}$, при которых минимизируется полная энергия $W$, равны 0.785 и 32.95 соответственно.

Предположим далее, что в момент времени $\tau=\tau_{O N}$ электрическое поле будет выключено, т. е. $E=0$ (turn-off процесс). В этом случае поле директора $\hat{\mathbf{n}}(x, z, \tau)$ под действием магнитных, вязких, упругих и поверхностных сил и моментов, действующих на единицу объема ЖК-фазы, начинает переориентироваться из состояния, характеризующегося углом $\theta_{e q}(x, z)$, в состояние, характеризующееся планарной ориентацией ЖК-ячейки. При этом угол $\theta(x, z, \tau)$ должен стремиться к нулю. На рис. 5 представлены результаты расчета эволюции угла $\theta(x, z=0, \tau)$ вдоль оси $x \in[-10,10]$ в течение $\tau=20$ единиц безразмерного времени, отсчитанного с момента выключения электрического поля. Была прослежена эволюция угла $\theta(\tau)$ от его начального распределения $\theta_{e q}^{(1)}(x, z=0)$ до конечного $\theta_{e q}=0$. Результат расчетов указывает на то, что быстрее релаксируют области, удаленные от положений узлов периодической структуры, т. е. $x= \pm 2.6, \pm 5.3$ и \pm 9.93 . Безразмерное время релаксации $\tau_{\mathrm{OFF}}$ поля директора к планарно ориентированному распределению по всему объему ЖК-ячейки равно 24 единицам безразмерного времени, или $\sim 31.2 \mathrm{~ms}$.

Располагая зависимостью угла $\theta(x, z, \tau)$ от времени $\tau$ и используя уравнения $(1)-(5)$, можно рассчитать эволюцию дейтеронного ЯМР-спектра $I(v)$ в зависимости от частоты $v$. На рис. 6 представлены результаты расчета

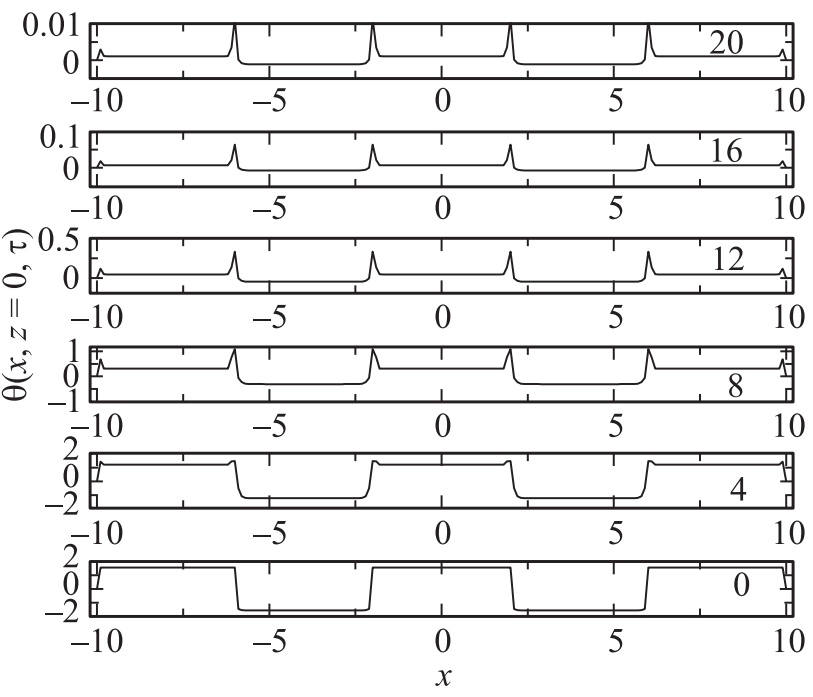

Рис. 5. Эволюция распределения угла $\theta(x, z=0, \tau)$ вдоль оси $x \in[-10,10]$ для случая $E=0$. Представлены состояния, соответствующие временам $\tau=0,4,8,12,16$ и 20.

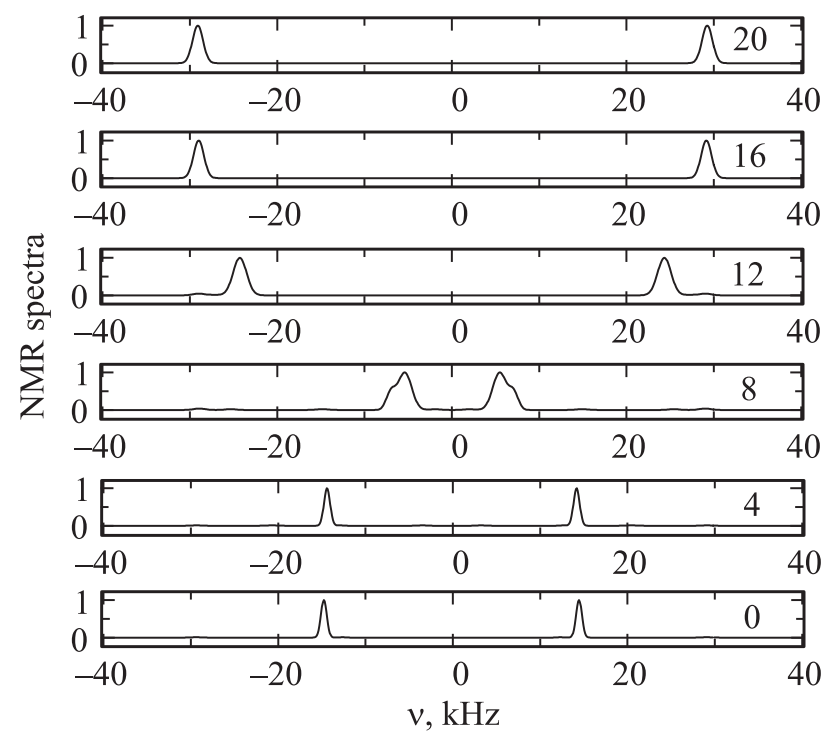

Рис. 6. Эволюция дейтеронного ЯМР-спектра $I(v)$ для turn-off процесса при температуре $300 \mathrm{~K}$, рассчитанная с помощью уравнения (1). 


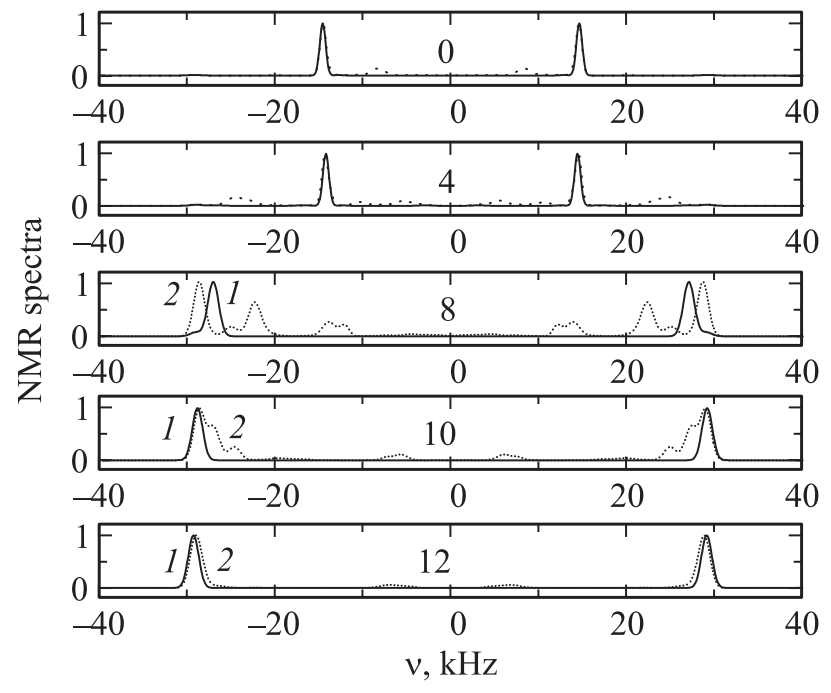

Рис. 7. Эволюция дейтеронного ЯМР-спектра $I(v)$ для turn-off процесса, полученная численными методами (сплошные кривые) и методами ЯМР-спектроскопии (штриховые кривые), для первых 12 единиц безразмерного времени, отсчитанного с момента выключения электрического поля.

функции $I(v)$, соответствующие ЖК-фазе образованной молекулами $5 Ц Б-d_{2}$, при температуре $300 \mathrm{~K}$, в процессе переориентации поля директора из положения, совпадающего с направлением электрического поля $\mathbf{E}$ $(U=200 \mathrm{~V})$, в положение, совпадающее с направлением магнитного поля $\mathbf{B}(B=7.08 \mathrm{~T})$. Представлены состояния, соответствующие безразмерным временам $\tau=0$ $\left(\tau=\tau_{O N}^{(1)}\right), 4,8,12,16$ и 20 после выключения электрического поля в момент времени $\tau=\tau_{O N}^{(1)}$. Значения параметров $\sigma_{2 L}(L=0,1,2)$, используемых при расчете ширины линии $\sigma(\theta)$, соответствовали значениям этих параметров, используемых в ЯМР-экспериментах [7], и были выбраны равными $\sigma_{0}=1.14, \sigma_{2}=0.523$ и $\sigma_{4}=-0.373 \mathrm{kHz}$, в то время как значение безразмерного параметра $\xi$ было выбрано равным $10^{4}$. Пределы изменения частоты $v \in[-40 \mathrm{kHz}, 40 \mathrm{kHz}]$ также соответствовали экспериментальным значениям [7]. Результат, представленный на рис. 6, свидетельствует о том, что выключение электрического поля $(E=0)$ приводит к уширению величины квадрупольного ЯМР-спектра $\Delta \bar{\nu}$ в 2 раза относительно $\Delta \bar{\nu}_{0}$ за время $\tau \sim 24(\sim 31.2 \mathrm{~ms})$. На рис. 7 представлены результаты сравнения эволюции дейтеронного ЯМР-спектра $I(v)$ (сплошные кривые), полученного численными методами с результатами, полученными с помощью ЯМР-спектроскопии (штриховые кривые), выполненные для случая 5ЦБ- $d_{2}$ и вышеописанных термодинамических условий. Эти результаты соответствуют первым 12 единицам безразмерного времени, отсчитанного с момента выключения электрического поля. Наблюдаемая тенденция к уширению величины расщепления квадрупольного спектра $\Delta \bar{\nu}$ в два раза относительно $\Delta \bar{\nu}_{0}$, свидетельствует о том, что поле директора переориентируется в положение, совпадающее с направлением магнитного поля $\mathbf{B}$.

\section{4. Заключение}

В предлагаемой работе представлено исследование процесса переориентации поля директора $\hat{\mathbf{n}}$ и эволюции ЯМР-спектра $I(v)$ нематического жидкого кристалла (ЖК), образованного молекулами дейтерированного 4-n-пентил-4'-цианобифенила, инкапсулированного в прямоугольную ЖК-ячейку, под действием сильных скрещенных электрического $\mathbf{E}$ и магнитного В полей, направленных под углом $\alpha$ друг к другу. Численные расчеты, выполненные в рамках нелинейного обобщения классической теории Эриксена-Лесли, показали, что при определенных соотношениях сил и моментов, действующих на единицу объема ЖК-фазы, в процессе переориентации $\hat{\mathbf{n}}$ могут возникнуть переходные периодические структуры, если соответствующая мода искажения обладает наибыстрейшим откликом и таким образом подавляет все остальные моды, в том числе и однородные. Показано, что возникновение этих периодических структур ведет к уменьшению времени переориентации поля директора. Так же изучена эволюция дейтеронного ЯМР-спектра $I(v)$, соответствующая процессу переориентации $\hat{\mathbf{n}}$ из состояния, направленного вдоль электрического поля $\mathbf{E}$, в состояние, направленное вдоль магнитного поля В (turn-off процесс). Это достигалось тем, что ЖК-образец, вначале сориентированный под действием сильных скрещенных электрического $\mathbf{E}$ и магнитного $\mathbf{B}$ полей, направленных под углом $\alpha\left(\sim \frac{\pi}{2}\right)$ друг к другу, с соответствующим расщеплением квадрупольного спектра $\Delta \bar{\nu}_{0}$ в какой-то момент времени $\tau=\tau_{O N}$, когда выключалось сильное поперечное электрическое поле $\mathbf{E}$ (turn-on процесс), начинает переориентироваться в направлении вектора $\mathbf{B}$. Эта переориентация ведет к уширению величины расщепления квадрупольного спектра $\Delta \bar{v}$ в 2 раза относительно $\Delta \bar{\nu}_{0}$. Сравнительный анализ поведения расчетных и экспериментальных результатов для ЯМР-спектров показал, что предложенная теоретическая модель, описывающая процесс переориентации $\hat{\mathbf{n}}$ и допускающая формирование переходных периодических структур, проливает свет на неизученные аспекты динамики поля директора в микроскопических ЖК-ячейках под действием скрещенных электрического и магнитного полей.

\section{Список литературы}

[1] P.G. de Gennes, J. Prost. The physics of liquid crystals. Oxford Univ. Press., Oxford (1995). 400 p.

[2] A.G. Chmielewski. Mol. Phys. Liq. Phys. 132, 319 (1986).

[3] R.F. Bruinsma, C.R. Safinya. Phys. Rev. A 43, 5377 (1991).

[4] A.V. Zakharov, A.A. Vakulenko, J. Thoen. J. Chem. Phys. 118, 425 (2003)

[5] D. Kamada, K. Okumoto, A. Sugimura, G.R. Luckhurst, B.A. Timimi, H. Zimmermann. Mol. Cryst. Liq. Cryst. 441, 131 (2005). 
[6] A.V. Zakharov, A.A. Vakulenko. J. Chem. Phys. 139, 244904 (2013).

[7] A. Sugimura, G.R. Luckhurst. Prog. Nucl. Mag. Res. Spectr. 94-95, 37 (2016).

[8] J.L. Ericksen. Arch. Ration. Mech. Anal. 4, 231 (1960).

[9] F.M. Leslie. Arch. Ration. Mech. Anal. 28, 265 (1968).

[10] S.M. Fan, G.R. Luckhurst, S.J. Picken. J. Chem. Phys. 101, 3255 (1994).

[11] А.В. Захаров, А.А. Вакуленко, С.В. Пасечник. ФТТ 58, 1851 (2016).

[12] A. Sugimura, A.V. Zakharov. Phys. Rev. E 84, 021703 (2011).

[13] A.V. Zakharov, A. Maliniak. Eur. Phys. J. E 4, 85 (2001).

[14] A.V. Zakharov, A. Maliniak. Eur. Phys. J. E 4, 321 (2001).

[15] А.В. Захаров, А.А. Вакуленко. Кристаллография 48, 738 (2003).

[16] И.С. Березин, Н.Р. Жидков. Методы вычислений. Физматгиз, М. (1964). 464 с. 